\title{
Adhering to Ethical Benchmarks in Neurology Clinical Trials Using iPSCs
}

\author{
Akira Akabayashi $^{1,2}$ (D) Eisuke Nakazawa ${ }^{1}$. Nancy S. Jecker ${ }^{3,4}$ \\ Published online: 28 March 2019 \\ (C) The American Society for Experimental NeuroTherapeutics, Inc. 2019
}

\begin{abstract}
We examine the ethics of using induced pluripotent stem cells (iPSCs) in cell transplantation treatment of neurologic diseases and the essential types of ethical benchmarks required in clinical trials in neurology using iPSCs, including embryonic pluripotent stem cells. We focus on two issues: (1) comparison and (2) criticism of the two types of neuro-hype (neuro-purism and neuroessentialism). In order to ensure that the dialog on ethical benchmarks continues to develop in a manner that promotes trust with society and research subjects, concerns about the clinical use of pluripotent stem cells (particularly iPSCs) in neurology must be at the forefront of any ethics discussion.
\end{abstract}

Keywords Research ethics $\cdot$ Neuroethics $\cdot$ iPS $\cdot$ Stem cell research $\cdot$ Bodily integrity $\cdot$ Personal identity

On November 9, 2018, Kyoto University Hospital announced that a team had performed a physician-initiated first-in-human clinical trial for Parkinson's disease (PD) involving the transplantation of dopaminergic progenitors generated from human induced pluripotent stem cells (iPSCs) [1]. On February 28, 2019, the specialist committee of the Ministry of Health, Labour and Welfare approved the protocol for the transplantation of iPSC-derived motor neurons in four patients with spinal cord injuries [2]. Meanwhile, on October 3, 2018, a team from Keio University published a paper demonstrating that a voltage-gated calcium channel antagonist benidipine, an FDA-approved drug library, suppresses rotenone-induced

Electronic supplementary material The online version of this article (https://doi.org/10.1007/s13311-019-00728-1) contains supplementary material, which is available to authorized users.

Akira Akabayashi

akirasan-tky@umin.ac.jp

1 Department of Biomedical Ethics, Faculty of Medicine, The University of Tokyo, 7-3-1 Hongo, Bunkyo-ku, Tokyo 113-0033, Japan

2 Division of Medical Ethics, New York University School of Medicine, New York, NY 10016, USA

3 Department of Bioethics and Humanities, University of Washington School of Medicine, Seattle, WA 98195-7120, USA

4 African Centre for Epistemology and Philosophy of Science, University of Johannesburg, Johannesburg, South Africa apoptosis using PD patient-specific iPSCs [3]. The two types of clinical application for iPSC use are drug discovery and cell transplantation. The present report examines the ethics of iPSC use in cell transplantation treatment of neurologic diseases; the examination was conducted broadly, including embryonic pluripotent stem cells (ESCs) as well as the types of ethical benchmarks that require adherence in treatment trials using iPSCs in neurology. We highlight two urgent issues: the comparison and the criticism of the two types of neuro-hype.

\section{Comparison}

\section{Uncertainty of iPSCs in Clinical Applications}

Due to the incomplete reprogramming of iPSCs, the epigenome and epigenetics of original cells are partially preserved. Consequently, cellular characteristics, including epigenetic characteristics, fluctuate widely by iPSC line. This makes it difficult to ensure reliable quality and raises the possibility of unforeseen events occurring. Moreover, iPSC lines inherit all somatic genome mutations.

Because the epigenome of the early embryonic cell is similar to that of pluripotent stem cells, fluctuations in the ESC epigenome are minor. Furthermore, ESC lines derived from germline cells that carry the genome to the next generation suppress genomic mutations. By contrast, iPSCs inherit differentiated cell genomes that have accumulated somatic 
mutations; as such, these will naturally have more genome mutations.

Neural stem cells, which already have been programmed to differentiate into neurons, have a limited capacity to propagate. Among them, fetal brain-derived neural stem cells, which have high propagation potential, face a limited ability to propagate in culture.

If iPSCs are chosen for cell transplantation treatment, overcoming the inherent quality issues discussed above will be important. By contrast, ESCs seem to be considered equivalent or better [4]. At the very least, comparative studies should be conducted between ESCs and iPSCs.

\section{Irreversibility and Aberrant Neural Reorganization}

Unlike retina [5] or heart [6] clinical trials, once iPSCs (or ESCs) are transplanted into the central nervous system (CNS) (which includes the brain and spinal cord), removing them is nearly impossible if the cells are rejected or if canceration occurs. This differs from the retina or heart, where the rejected portion can be removed by funduscopy or surgery. In the case of the CNS, the only options are irradiation or surgical removal of the transplanted region, which might irreparably damage large regions. Depending on the targeted region, this might reduce the QOL relative to that before transplantation.

One of our major concerns is the possibility for pluripotent stem cells to promote aberrant neural reorganization when cell transplantation treatment is used. As Bretzner et al. reported for cases of spinal cord injury [7], "the presence of either undifferentiated hESCs (human embryonic stem cells) and/ or inappropriate inflammation may trigger aberrant changes to central nervous system networks that could lead to neurologic dysfunction such as hyperreflexia, spasticity, dystonia, pain, and allodynia."

The command chain from the CNS to peripheral systems represents an extraordinarily intricate network that controls a delicate balance in order to maintain homeostasis throughout the body. In other words, the CNS is unlike other localized tissue or organs which do not directly impact other organs. This characteristic warrants ethical review. Indeed, there is always the possibility that transplanting cells into the CNS could disrupt the delicate balance of the overall network.

Besides the local graft-induced dyskinesia, this possibility should be carefully considered and observed when pluripotent stem cells are transplanted into CNS areas.

Irreversibility is tied to the issue of informed consent. Many neurology patients lack the capacity to provide consent (e.g., due to advanced stage Alzheimer's disease) and some are children with hereditary disorders. If treatment for such patients is fundamentally premised on consent by proxy, then careful ethical scrutiny is required, because once initiated, these treatments cannot be reversed. The situation differs from that of the retina and heart, where transplanted tissue could simply be removed if the subject decides to stop trial participation. It may be best to erect a more conscientious framework for handling the conditions of proxy consent and respecting the individual's right to refuse. This is particularly important when using iPSCs in the CNS, given the greater possibility of unknown effects compared with other stem cells applications.

\section{Autologous or Allogeneic iPSCs?}

In the planned Parkinson's disease (PD) study, iPS stock cells will be used. iPS stock cells are allogeneic cells generated from individuals with HLA types with a lower likelihood of rejection [8]. They are still cells from another person, however, and immune-suppressants are required, which increases patient burden. In the case of PD, autologous iPSCs have a genetic background which leads synuclein to aggregate into Lewy bodies, and thus, allogeneic iPSCs may be considered better options. According to this hypothesis, it is better to use allogeneic iPSCs in sporadic diseases, such as sporadic amyotrophic lateral sclerosis. In the case of sporadic diseases, risks of immune-suppressant should be balanced with this possibility, although, there is so far no strong scientific evidence [9]. At the very least, these two types of cells should be carefully compared in terms of efficacy and risks on a case-by-case basis.

\section{Criticism of Two Types of Neuro-Hype}

Reorganization of the neural network due to transplantation of pluripotent stem cells to the CNS requires neuroethical consideration. From at least two perspectives, reorganization of neural networks may evoke ethical concerns in the general public. However, we believe that this may actually be a type of neuro-hype.

\section{Neuro-Purism}

One form of neuro-hype is "neuro-purism." The neuro-purism to which we refer emphasizes physical integrity and pureness. For example, in xenotransplantation, and even in allogeneic organ transplantation (e.g., heart, lung, liver, and kidney), engraftment of another person's organs into one's own body might be viewed by society with apprehension, as a threat to bodily integrity or personal identity. Bodily integrity is valuable to human beings and delineating between self and others is integral to personal identity. For this reason, it is not simple to justify this type of neuro-purism. A powerful counterargument that must be addressed is that even if people hold that bodily integrity is compromised through xenotransplantation or allogeneic organ transplantation, this is a temporary 
psychological repugnance toward novelty that will diminish as people become accustomed and adjust.

\section{Neuro-Essentialism}

The other form of neuro-hype is "neuro-essentialism"; in the present context, this approach holds that the brain is a special organ and that the brain alone is responsible for individual personality. From that perspective, the CNS might be considered the seat of personal identity. Therefore, the reorganization of the neural network through transplantation of stem cells to the CNS may, at first glance, seem like an affront to personal identity.

In response, however, such an interpretation conflates two distinct aspects of personal identity: numerical and qualitative. If something persists throughout a period of time as one and the same thing, it is numerically identical. If two things are qualitatively identical, they share the same property. For example, two oranges are numerically different but qualitatively identical: they share the same property of being oranges, although they are two distinct oranges, not one. We can apply this categorization to the concept of personal identity as well. Our present characters are different from those when we were 10 years old. However, both belong to our identical lives, which is why we have numerical identity.

Under normal conditions, it is rare that numerical identity becomes unstable or threatened. As a practical matter, our lives are constructed on the basis of personal numerical identities. If the reorganization of the neural network through stem cell transplantation to the CNS threatens the integrity of someone's numerical identity, this raises a red flag, suggesting perhaps that it should be regulated. Reorganization of the neural network through stem cell transplantation to the CNS can also threaten qualitative identity and our sense of a unified self. With respect to qualitative identity, one approach is to assess whether change occurs rapidly or slowly. Rapidly altering might be more prone than slow altering to disrupt a person's sense of a unified self. In the ordinary case, the pace at which personal qualities comprising qualitative identity transform is slow. We can form relatively stable and unified selfconceptions even though we change gradually over time.

As far as determining what kinds of characteristics are embodied through the reorganization of the neural network through stem cell transplantation to the CNS, particularly when compared to changes to character and belief systems as a result of administration of psychotropic drugs, deep brain stimulation, or other neurosurgeries, further experience with these techniques will be needed. Of course, the changes are dependent on the area of the brain targeted by these transplantations. We propose that transplantation into the areas of the brain that control moral judgments and emotions requires utmost care and consideration.

If the above concerns are held at the forefront of discussions on ethical benchmarks, the clinical use of pluripotent stem cells in neurology, particularly iPSCs, may very well continue to progress in a manner that maintains society's trust.

Required Author Forms Disclosure forms provided by the authors are available with the online version of this article.

\section{Compliance with Ethical Standards}

Competing Interests AA is the President of the Japan Association for Bioethics; this paper reflects the author's personal academic analyses and opinions and does not represent JAB's official position. EN and NSJ have no conflicts of interest to declare.

\section{References}

1. Center for iPS Cell Research and Application (CiRA) News. Announcement of physician-initiated clinical trials for Parkinson's disease. http://www.cira.kyoto-u.ac.jp/e/pressrelease/news/180730170000.html. Accessed 24 March 2019.

2. Nikkei Shimbun. iPS treatment: core of 'regenerative medicine,' challenge for recovery from spinal cord injury: Keio University protocol has been approved. February 19, 2019.

3. Tabata Y et al. T-type calcium channels determine the vulnerability of dopaminergic neurons to mitochondrial stress in familial Parkinson disease. Stem Cell Reports 2018; 11(5):1171-1184. https://doi.org/ 10.1016/j.stemcr.2018.09.006.

4. Doi D, Morizane A, Kikuchi T, et al. Prolonged maturation culture favors a reduction in the tumorigenicity and the dopaminergic function of human ESC-derived neural cells in a primate model of Parkinson's disease. Stem Cells 2012; 30: 935-945.

5. Mandai M, Watanabe A, Kurimoto Y, et al. Autologous induced stem-cell-derived retinal cells for macular degeneration. N Engl J Med 2017; 376:1038-1046.

6. Cyranoski D. Reprogrammed stem cells approved to mend hearts. Nature 2018; 557: 619-620.

7. Bretzner F, Gilbert F, Baylis F, Brownstone RM. Target populations for first-in-human embryonic stem cell research in spinal cord injury. Cell Stem Cell 2011 8(5):468-75.

8. Akabayashi A, Nakazawa E, Jecker NS. Endangerment of the iPSC stock project in Japan: on the ethics of public funding policies. Journal of Medical Ethics 2018; 44: 700-702.

9. Morizane A, Doi D, Kikuchi T, et al. Direct comparison of autologous and allogeneic transplantation of iPSC-derived neural cells in the brain of a nonhuman primates. Stem Cell Reports 2013; 1: 283292.

Publisher's Note Springer Nature remains neutral with regard to jurisdictional claims in published maps and institutional affiliations. 